\title{
The Importance of G-protein Coupled Estrogen Receptor in Patients With Fibromyalgia
}

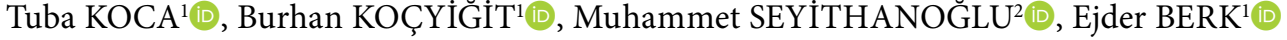 \\ ${ }^{1}$ Department of Physical Medicine and Rehabilitation, Kahramanmaraş Sütçü İmam University Faculty of Medicine, Kahramanmaraş, Turkey \\ ${ }^{2}$ Department of Clinic Biochemistry, Kahramanmaraş Sütçü Imam University Faculty of Medicine, Kahramanmaraş, Turkey
}

\begin{abstract}
Objectives: This study aims to analyze the G-protein coupled estrogen receptor (GPER/GPR30) activity in patients with fibromyalgia syndrome (FMS).

Patients and methods: We enrolled 40 female patients with FMS (mean age $42.9 \pm 11.2$ years; range, 18 to 64 years) diagnosed according to the 2010 American College of Rheumatology classification criteria and 30 age- and body mass index-matched female healthy controls (mean age $43.7 \pm 13.6$ years; range, 19 to 64 years). Sex hormones of patients (morning) including estradiol, follicle stimulating hormone, luteinizing hormone, and prolactin (PRL) were recorded. FMS severity was assessed by Fibromyalgia Impact Questionnaire (FIQ). Serum GPER levels were measured by using a quantitative sandwich enzyme-linked immunosorbent assay method with a commercial kit.

Results: G-protein coupled estrogen receptor levels were $0.11(0.02-0.9) \mathrm{ng} / \mathrm{mL}$ in the FMS patients and $0.059(0.01-0.13) \mathrm{ng} / \mathrm{mL}$ in controls, with a statistically significant difference $(p=0.037)$. GPER levels were positively correlated with age and negatively correlated with PRL, while they were not correlated with FIQ. Differential diagnosis for FMS with receiver operating characteristic (ROC) analysis for the serum GPER levels was statistically significant (area under the ROC curve: 0.653 , confidence interval: $0.522-0.785, p=0.029$ ). High values indicated FMS, with a threshold of $>0.075$, sensitivity of $60 \%$, and specificity of $60 \%$.
\end{abstract}

Conclusion: The GPER levels of FMS patients were higher than those of the controls. Thus, GPER levels may be considered as a biomarker in the diagnosis of FMS independent of disease severity.

Keywords: Estrogen; fibromyalgia; G-protein coupled estrogen receptor; sex hormone.

Fibromyalgia syndrome (FMS) is a complex syndrome characterized by chronic pain, fatigue, and dysfunctional symptoms. The etiology of FMS is still controversial. Genetic susceptibility, environmental triggers, and neuromodulation affect the onset and duration of the disease. ${ }^{1}$ The common symptoms of FMS include sleep disorders, affective disorders, chronic generalized pain, and fatigue. The pathophysiology of FMS has not been elucidated yet, and no treatment is available for relieving all of the symptoms. The prevalence of FMS in females is explained by increased symptoms in sex hormone-related events such as premenstrual, pregnancy, postpartum, and postmenopausal. ${ }^{2}$ It has a significant impact on the social life and quality of life in the areas of the ability to go to work, participate in daily life, entertainment, and sporting activities. Treatment includes medication such as analgesics, tricyclic antidepressants, cognitive behavioral therapy, exercises, and training programs.

Hormonal fluctuations occur in FMS patients due to chronic pain and stress. Many studies

Received: October 15, 2018 Accepted: December 03, 2018 Published online: January 23, 2019

Correspondence: Tuba Koca, MD. Kahramanmaraş Sütçü İmam Üniversitesi Tıp Fakültesi Fiziksel Tıp ve Rehabilitasyon Anabilim Dalı, 46040, Onikişubat, Kahramanmaraş, Turkey. Tel: +90344-228 2800 e-mail: tuba_baglan@yahoo.com

\section{Citation:}

Koca T, Koçyiğit B, Seyithanoğlu M, Berk E. The importance of G-protein coupled estrogen receptor in patients with fibromyalgia. Arch Rheumatol 2019;34(4):419-425. 
have revealed hypothalamo-pituitary-adrenal axis dysfunction hypothesis. It is thought that hypothalamic corticotropin-releasing hormone (CRH) neurons are active in the central nervous system in association with chronic stress. This activation results in irregularities in the release of gonadotropins and cortisol hormone. ${ }^{1,2}$

Estrogen plays an important role in normal physiology, aging, and regulation of many diseases. Several endogenous estrogens, particularly estradiol (E2), are known to mediate adverse effects that may affect puberty, reproductive, metabolic, endocrinological, musculoskeletal, nervous, cardiovascular, and immunological systems. ${ }^{3,4}$ Sex hormones, particularly estrogens, have important effects on pain duration and sensitivity (hyperalgesia), while estrogen deficiency is considered as a risk factor for the development of FMS.5,6 There are few studies investigating the role of estrogen in the FMS treatment. Blümel et al. ${ }^{7}$ have demonstrated epidemiological, etiopathogenic, symptomatic, and therapeutic similarities between FMS and climacteric syndrome. It has been observed that some symptoms reverse after hormone replacement therapy. ${ }^{7}$

Recent studies have shown that G-protein coupled estrogen receptor (GPER/GPR30) binds to estrogen directly and mediates its activity. It was shown to be a therapeutic target and antagonist, also a prognostic indicator in some diseases. ${ }^{8,9}$ Therefore, in this study, we aimed to analyze the GPER/GPR30 activity in patients with FMS.

\section{PATIENTS AND METHODS}

This single-centered prospective case-control study was conducted at Kahramanmaraş Sütçü İmam University Faculty of Medicine between January 2018and April 2018 and included 40 female patients with FMS (mean age $42.9 \pm 11.2$ years; range, 18 to 64 years) diagnosed according to the 2010 American College of Rheumatology classification criteria and 30 age- and body mass index-(BMI) matched healthy controls (mean age $43.7 \pm 13.6$ years; range, 19 to 64 years). Age, height, weight, sex hormones (morning) including $\mathrm{E} 2$, follicle stimulating hormone (FSH), luteinizing hormone (LH), and prolactin (PRL) were recorded. The study protocol was approved by the Kahramanmaraş Sütçü İmam University Faculty of Medicine Ethics Committee (protocol no: 2017/145). A written informed consent was obtained from each participant. The study was conducted in accordance with the principles of the Declaration of Helsinki.

Those with a history of hormone replacement therapy, endocrinological disorder, gynecologic or other malignancies, active infection or inflammatory rheumatological disease were excluded. The Fibromyalgia Impact Questionnaire (FIQ) was used to evaluate patients' functional status, disease progression, and outcomes. The Turkish validity study of the FIQ was performed by Sarmer et al. ${ }^{10}$ The first item of the scale consists of 10 Likert-type questions. In the second and third items, patients are asked to select the days to allow for the determination of "disease exposure" and "absence from work." The scores obtained are adapted to 10 . The remaining seven questions are based on marking the corresponding points in the equivalent visual scale. The score interval is $0-100$.

Venous blood samples were obtained from the antecubital area by phlebotomy. After these samples were allowed to clot, they were centrifuged for 10 minutes at $4,000 \mathrm{rpm}$ $(4,000 \mathrm{rpm}$ corresponds to $1,610 \mathrm{~g}$ and it is the most appropriate technique). The sera were separated and stored at $-80^{\circ} \mathrm{C}$ until the analysis. This temperature is the most appropriate level of heat where molecules are at the most stable situation.

Serum FSH, PRL, and LH were measured by using a Centaur XP Immunoassay System (Siemens Healthcare GmbH, Erlangen, Germany). Serum GPER (SEG045Hu, Cloud-CloneCorp., Houston, Texas, USA) levels were measured using the commercial enzyme-linked immunosorbent assay kits according to the manufacturer's instructions. Since the majority of the values were below the limit of sensitivity of the kit $(0.125 \mathrm{ng} / \mathrm{mL})$, the concentration was detected by calculation. The margin of error of the measurement is not known as it is rarely used for daily routine.

\section{Statistical analysis}

Statistical analyses were applied using the IBM SPSS version 22.0 (IBM Corp., Armonk, NY, USA). Descriptive statistics were stated 
Table 1. Descriptive and analytic characteristics of groups

\begin{tabular}{|c|c|c|c|c|c|c|c|}
\hline & \multicolumn{3}{|c|}{ Fibromyalgia syndrome group $(n=40)$} & \multicolumn{3}{|c|}{ Control group $(n=30)$} & \multirow[b]{2}{*}{$p$} \\
\hline & Mean $\pm \mathrm{SD}$ & Median & Min-Max & Mean \pm SD & Median & Min-Max & \\
\hline Age (year) & $42.9 \pm 11.2$ & & & $43.7 \pm 13.6$ & & & 0.66 \\
\hline Body mass index $\left(\mathrm{kg} / \mathrm{m}^{2}\right)$ & $30.9 \pm 4.9$ & & & $28.6 \pm 7.9$ & & & 0.32 \\
\hline Fibromyalgia Impact Questionnaire & $56.3 \pm 2.3$ & & & - & & & \\
\hline G-protein coupled estrogen receptor $(\mathrm{ng} / \mathrm{mL})^{*}$ & & 0.11 & $0.02-0.9$ & & 0.059 & $0.01-0.13$ & 0.037 \\
\hline Estradiol $(\mathrm{pg} / \mathrm{mL})$ & & 68.5 & $11-213$ & & 67.6 & $12-200$ & 0.068 \\
\hline Follicle stimulating hormone $(\mathrm{mU} / \mathrm{mL})$ & & 30.1 & $80-117$ & & 29.6 & $65-110$ & 0.42 \\
\hline Luteinizing hormone $(\mathrm{mU} / \mathrm{mL})$ & & 19.1 & $20-76$ & & 24.2 & $11-56$ & 0.12 \\
\hline Prolactin (ng/mL) & & 8.2 & $2-24.7$ & & 9.2 & $4.4-12.2$ & 0.23 \\
\hline
\end{tabular}

SD: Standard deviation; Min-max: Minimum-maximum; Independent samples t-test was used for age, body mass index, and Fibromyalgia Impact Questionnaire. Mann-Whitney U test was used for G-protein coupled estrogen receptor and sex hormones. " A p value of $<0.05$ was accepted as statistically significant.

as median, minimum/maximum and mean \pm standard deviation values. Conformity of the data to normal distribution was evaluated with the Kolmogorov-Smirnov test. In the comparison of groups not showing normal distribution, the Mann-Whitney $U$ test was used. Independent samples (Student's) t-test was used in normally distributed parameters. Correlations between the variables were examined with Spearman's test. For diagnostic tests of GPER-1, the receiver operating characteristic (ROC) curve was applied and the threshold value for GPER was manually selected according to both highest sensitivity and highest 1 - specificity. A $p$ value of $<0.05$ was accepted as statistically significant.

\section{RESULTS}

Mean ages of FMS patients and controls were $42.9 \pm 11.2$ years and $43.7 \pm 13.6$ years, respectively $(p=0.66)$. Mean BMIs of FMS patients and controls were $30.9 \pm 4.9 \mathrm{~kg} / \mathrm{m}^{2}$ and $28.6 \pm 7.9 \mathrm{~kg} / \mathrm{m}^{2}$, respectively $(\mathrm{p}=0.32)$. The median E2 levels of FMS patients and controls were $68.5 \mathrm{pg} / \mathrm{mL}$ and $67.6 \mathrm{pg} / \mathrm{mL}$, respectively $(p=0.068)$. The median FSH levels of FMS patients and controls were $30.1 \mathrm{mU} / \mathrm{mL}$ and $29.6 \mathrm{mU} / \mathrm{mL}$, respectively $(\mathrm{p}=0.42)$. The median LH levels of FMS patients and controls were $19.1 \mathrm{mU} / \mathrm{mL}$ and $24.2 \mathrm{mU} / \mathrm{mL}$, respectively $(p=0.12)$. The median PRL levels of FMS patients and controls were $8.2 \mathrm{ng} / \mathrm{mL}$ and $9.2 \mathrm{ng} / \mathrm{mL}$, respectively $(p=0.23)$. The descriptive and analytic data for the groups were summarized in Table 1.
G-protein coupled estrogen receptor levels of FMS patients and controls were 0.11 $(0.02-0.9) \mathrm{ng} / \mathrm{mL}$ and 0.059 (0.01-0.13) ng/mL, respectively, and the difference was statistically significant ( $p=0.037)$ (Figure 1). In FMS patients, GPER was negatively correlated with PRL (correlation coefficient $[\mathrm{rho}]=-0.37 ; \mathrm{p}=0.03$ ) and positively correlated with age ( $\mathrm{rho}=0.41 ; \mathrm{p}=0.03$ ), while it was not correlated with BMI, FIQ, E2, FSH or LH levels.

Differential diagnosis for FMS with ROC analysis for the serum GPER levels was statistically significant (area under the ROC curve [AUC]: 0.653 [medium], confidence interval: 0.522-0.785, $\mathrm{p}=0.029$ ). High values indicated FMS, with a threshold of $>0.075$, sensitivity of $60 \%$, and specificity of $60 \%$ (Figure 2).

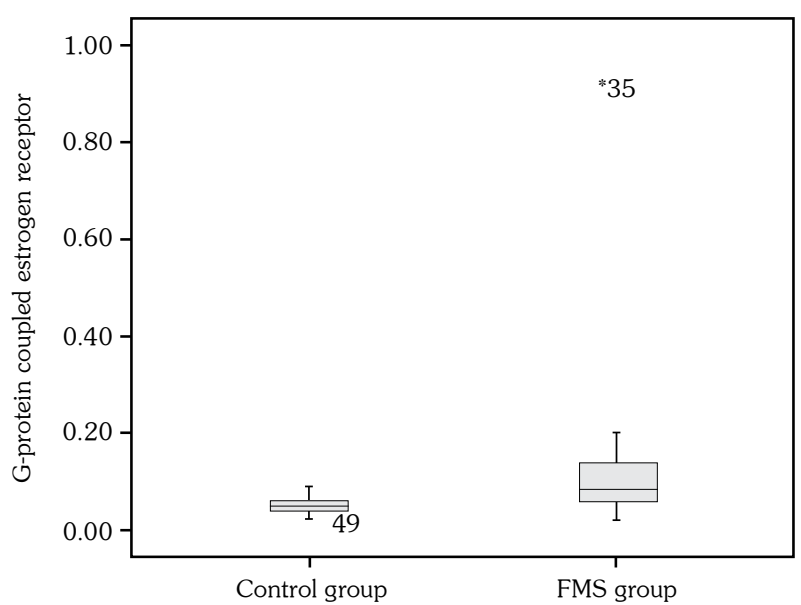

Figure 1. Boxplot of G-protein coupled estrogen receptor values of groups. FMS: Fibromyalgia syndrome. 


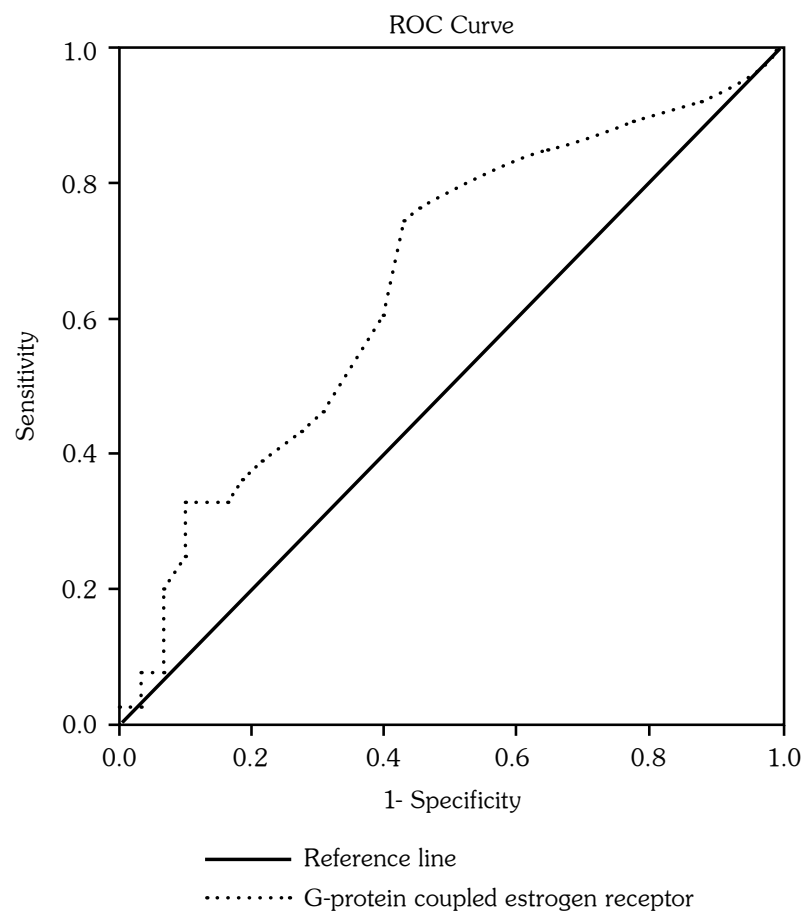

Figure 2. Receiver operating characteristic curve analysis of G-protein coupled estrogen receptor. ROC: Receiver operating characteristic.

\section{DISCUSSION}

Estrogen is an important metabolic syndrome regulator that includes abnormalities including obesity, insulin resistance/glucose intolerance, hypertension, dyslipidemia, and inflammation. ${ }^{11}$ GPER is widely expressed in both normal and pathological tissues in the human body. Recent studies have used a sandwich enzyme immunoassay kit for quantitative in vitro measurement of GPER levels in human serum, plasma, tissue homogenate, and other biological fluids. ${ }^{12,13}$

The GPER has now become recognized as a major mediator of estrogen's rapid cellular effects throughout the body. ${ }^{8,14}$ While it is known that GPER has seven membranes and non-genomic signal processing, its functions and mechanisms of physiological effects are not well known. ${ }^{15}$ It plays an important role in estrogen-mediated neuroprotection. In light of the current literature, GPER is thought to have role in hypertension, renal disease, atherosclerosis, vascular remodeling, heart failure, reproduction, metabolic disorders, cancer, and menopause. ${ }^{16-19}$ It was observed by Lu et al. ${ }^{18}$ that ischemic brain damage was reduced by activation of vascular endothelial growth factor A by GPER activation in rats. The presence of low prevalence of E2 is attributed to many diseases in premenopausal females. ${ }^{6}$

One of the important issues emphasized in FMS etiopathogenesis is hypothalamo-pituitarygonadal axis dysfunction. Hyperactivation of $\mathrm{CRH}$ neurons, decreased levels of serotonin and growth hormone, and reduced corticosteroid metabolites in urine were listed in the current data. Different results have been reported regarding changes in sex hormones. ${ }^{20-22}$ Increased levels of basal adrenocorticotropic hormone (ACTH), FSH and cortisol; decreased levels of insulin like growth factor-1 (somatostatin C), free triiodothyronine, and estrogen were detected. Systemic increase in $\mathrm{CRH}$, growth hormone-releasing hormone, thyrotropinreleasing hormone, and luteinizing hormonereleasing hormone result in increased $\mathrm{ACTH}$ and PRL levels and decreased thyroid-stimulating hormone. Hormonal changes in FMS patients can be explained by chronic pain-dependent primary stress activation of hypothalamic $\mathrm{CRH}$ neurons. ${ }^{20-23}$ Briefly, neuroendocrine disorders in FMS indicate gonadal steroid hormone abnormalities. ${ }^{24}$ In our study, sex hormones of FMS patients were found to be similar to those of controls.

The GPER is expressed throughout the central nervous system with highest levels of expression in the hypothalamo-pituitary axis, hippocampal formation, and brain stem autonomic nuclei. ${ }^{25,26}$ Recent evidence suggests that GPER may serve a neuroprotective effect. ${ }^{27}$ Fibromyalgia decreases the quality of life in pre- and postmenopausal females. Identification of the possible functions of GPER may guide us in identifying the sex-specific risks. According to our study, GPER levels of FMS patients were higher than those of controls regardless of disease severity and sex hormones. We have also revealed that GPER can be used as a moderate diagnostic test for FMS. We have found high GPER activity, with normal serum E2 in the study group. This may show us the disregulation of metabolic pathways associated with obesity, insulin resistance/glucose intolerance, hypertension, dyslipidemia, inflammation or neuroendocrine hormones, neurotransmitters and cytokines in FMS patients. Pharmacological stimulation of GPER in vivo limits weight gain and improves metabolic output, revealing a promising novel 
therapeutic potential for the treatment of obesity and diabetes. ${ }^{28}$ In our study, GPER was not correlated with BMI in FMS patients. It was suggested that GPER has a modulator role in energy homeostasis (weight control). ${ }^{29}$

In our study, GPER levels were positively correlated with age and negatively correlated with PRL. Increased GPER activity with age may be attributed to the decreased amount of E2 with advanced age. Camilletti et al. ${ }^{30}$ have found that local GPER expression is regulated negatively by $\mathrm{E} 2$, progesterone, and lack of ovarian steroids after an ovariectomy significantly increases pituitary GPER expression. They have also shown a rapid E2 stimulatory effect on PRL secretion mediated by GPER. ${ }^{30,31}$

The GPER mediates the rapid effects of estrogen and aldosterone. Current studies indicate that GPER activation regulates blood pressure. Feldman ${ }^{32}$ have shown that genetic hypofunction of GPER induces hypertension and hypercholesterolemia in females. Li et al. ${ }^{33}$ have revealed increased cardioprotective effect of GPER by increasing ischemia reperfusion and inhibiting cardiocyte apoptosis. ${ }^{34}$ Vasculary GPER activation is associated with vasodepressor effect in animals. GPER16L, the most common genetic trait, is hypofunctional, and these allele carriers have high blood pressure. Today's data support that females with impaired GPER function carry an increased risk of high blood pressure. ${ }^{35}$ Santollo et al. ${ }^{35}$ have found that GPER regulates fluid and nutrient uptake in rats. GPER was shown to have positive effects on vascular functions. ${ }^{36}$

Newly discovered roles of GPER are cognition, depression, homeostasis, pain processing, and other associated neuronal functions. Also, increased anxiety, depression or cognitive disorders related with GPER activity have been determined. ${ }^{37,38}$ Postmenopausal depression is associated with decreased ovarian hormones. It is believed that discrepancies regarding the nature of estrogens' effects on anxiety are attributable to the differential effects of specific estrogen receptor subtypes. ${ }^{39}$ It is not known which estrogen receptor plays a role by potentiating the brain mitochondrial function in the neuroprotection of estrogen. In our study, we have not examined FMS patients in terms of cognitive functions or depression. This may be the subject of future randomized controlled studies. ${ }^{31}$

This study has some limitations. Firstly, there are disagreements in the literature including some suggesting that GPER cannot appear in serum. Secondly, we have expected to find the E2 levels, together with levels of the other sex hormones, to be different in our FMS group. Beyond hypothalamo-pituitary-adrenal axis dysfunction, there may be other confounding factors affecting GPER activity in FMS patients. Furthermore, in the ROC curve analysis, AUC was $>0.6$, thus the test is moderate as a diagnostic test. Moreover, the small sample size and lack of considering other hormones such as cortisol, progesterone, or testosterone affecting the GPER activity are the other limitations of our study.

In conclusion, many protective effects of estrogens in females are well known. The role of estrogen and its activity on FMS pathogenesis, which often affects females in their reproductive period, should be the subject of new researches. Our study has shown high levels of GPER levels in FMS patients compared to controls. Therefore, GPER levels may be considered as a biomarker in the diagnosis of FMS independent of disease severity and sex hormones.

\section{Declaration of conflicting interests}

The authors declared no conflicts of interest with respect to the authorship and/or publication of this article.

\section{Funding}

The authors received no financial support for the research and/or authorship of this article.

\section{REFERENCES}

1. Talotta R, Bazzichi L, Di Franco M, Casale R, Batticciotto A, Gerardi MC, et al. One year in review 2017: fibromyalgia. Clin Exp Rheumatol 2017;35:6-12.

2. Hernandez-Leon A, De la Luz-Cuellar YE, GranadosSoto V, González-Trujano ME, Fernández-Guasti A. Sex differences and estradiol involvement in hyperalgesia and allodynia in an experimental model of fibromyalgia. Horm Behav 2018;97:39-46.

3. Sharma G, Prossnitz ER. GPER/GPR30 Knockout Mice: Effects of GPER on Metabolism. Methods Mol Biol 2016;1366:489-502. 
4. Kim MJ, Kim TH, Lee HH. G-protein Coupled Estrogen Receptor (GPER/GPR30) and Women's Health. J Menopausal Med 2015;21:79-81.

5. Stening KD, Eriksson O, Henriksson KG, Brynhildsen $J$, Lindh-Åstrand L, Berg G, et al. Hormonal replacement therapy does not affect self-estimated pain or experimental pain responses in post-menopausal women suffering from fibromyalgia: a double-blind, randomized, placebo-controlled trial. Rheumatology (Oxford) 2011;50:544-51.

6. Okifuji A, Turk DC. Sex hormones and pain in regularly menstruating women with fibromyalgia syndrome. J Pain 2006;7:851-9.

7. Blümel JE, Palacios S, Legorreta D, Vallejo MS, Sarra $\mathrm{S}$. Is fibromyalgia part of the climacteric syndrome? Maturitas 2012;73:87-93.

8. Prossnitz ER, Hathaway HJ. What have we learned about GPER function in physiology and disease from knockout mice? J Steroid Biochem Mol Biol 2015;153:114-26.

9. Cygankiewicz AI, Jacenik D, Krajewska WM. GPER receptor - the new player in estrogen signaling. Postepy Biochem. 2015;61(1):52-60.

10. Sarmer S, Ergin S, Yavuzer G. The validity and reliability of the Turkish version of the Fibromyalgia Impact Questionnaire. Rheumatol Int 2000;20:9-12.

11. Sharma G, Hu C, Brigman JL, Zhu G, Hathaway HJ, Prossnitz ER. GPER deficiency in male mice results in insulin resistance, dyslipidemia, and a proinflammatory state. Endocrinology 2013;154:4136-45.

12. Bilal N, Kurutas EB, Orhan I. An evaluation of G-protein coupled membrane estrogen receptor-1 level in stuttering. Eur Arch Otorhinolaryngol 2018;275:469-76.

13. Revankar CM, Cimino DF, Sklar LA, Arterburn JB, Prossnitz ER. A transmembrane intracellular estrogen receptor mediates rapid cell signaling. Science 2005;307:1625-30.

14. Prossnitz ER, Barton M. Estrogen biology: new insights into GPER function and clinical opportunities. Mol Cell Endocrinol 2014;389:71-83.

15. Sandén C, Broselid S, Cornmark L, Andersson $\mathrm{K}$, Daszkiewicz-Nilsson J, Mårtensson UE, et al. $G$ protein-coupled estrogen receptor $1 / G$ proteincoupled receptor 30 localizes in the plasma membrane and traffics intracellularly on cytokeratin intermediate filaments. Mol Pharmacol 2011;79:400-10.

16. Zimmerman MA, Budish RA, Kashyap S, Lindsey $\mathrm{SH}$. GPER-novel membrane oestrogen receptor. Clin Sci (Lond) 2016;130:1005-16.

17. Barton M, Prossnitz ER. Emerging roles of GPER in diabetes and atherosclerosis. Trends Endocrinol Metab 2015;26:185-92.

18. Lu D, Qu Y, Shi F, Feng D, Tao K, Gao G, et al. Activation of $G$ protein-coupled estrogen receptor 1 (GPER-1) ameliorates blood-brain barrier permeability after global cerebral ischemia in ovariectomized rats. Biochem Biophys Res Commun 2016;477:209-14.
19. Prossnitz ER, Barton M. The G-protein-coupled estrogen receptor GPER in health and disease. Nat Rev Endocrinol 2011;7:715-26.

20. Samborski W, Sobieska M, Pieta P, Drews K, Brzosko M. Normal profile of sex hormones in women with primary fibromyalgia. Ann Acad Med Stetin 2005;51:23-6.

21. Koca TT, Karaca Acet G, Tanrikut E, Talu B. Evaluation of sleep disorder and its effect on sexual dysfunction in patients with Fibromyalgia syndrome. Turk J Obstet Gynecol. 2016;13:167-71.

22. Tulay KT, Emrullah T, Aydin A, Ciledag OF. The effect of fibromyalgia syndrome to gravidity, parity and duration of breastfeeding; A prospective study from Turkey. Pak J Med Sci 2016;32:545-9.

23. Ostensen M, Rugelsjøen A, Wigers SH. The effect of reproductive events and alterations of sex hormone levels on the symptoms of fibromyalgia. Scand $\mathrm{J}$ Rheumatol 1997;26:355-60.

24. Korszun A, Young EA, Engleberg NC, Masterson L, Dawson EC, Spindler K, et al. Follicular phase hypothalamic-pituitary-gonadal axis function in women with fibromyalgia and chronic fatigue syndrome. J Rheumatol 2000;27:1526-30.

25. Brailoiu E, Dun SL, Brailoiu GC, Mizuo K, Sklar LA, Oprea TI, et al. Distribution and characterization of estrogen receptor $G$ protein-coupled receptor 30 in the rat central nervous system. $J$ Endocrinol 2007;193:311-21.

26. Naugle MM, Gore AC. GnRH neurons of young and aged female rhesus monkeys co-express GPER but are unaffected by long-term hormone replacement. Neuroendocrinology 2014;100:334-46.

27. Lebesgue $\mathrm{D}$, Traub M, De Butte-Smith M, Chen C, Zukin RS, Kelly MJ, et al. Acute administration of non-classical estrogen receptor agonists attenuates ischemia-induced hippocampal neuron loss in middleaged female rats. PLoS One 2010;5:8642.

28. Sharma G, Prossnitz ER. G-Protein-Coupled Estrogen Receptor (GPER) and Sex-Specific Metabolic Homeostasis. Adv Exp Med Biol 2017;1043:427-53.

29. Davis KE, Carstens EJ,, Irani BG, Gent LM, Hahner LM, Clegg DJ. Sexually dimorphic role of G proteincoupled estrogen receptor (GPER) in modulating energy homeostasis. Horm Behav 2014;66:196-207.

30. Camilletti MA, Abeledo Machado AI, Ferraris J, Pérez PA, Faraoni EY, Pisera D, et al. Role of GPER in the anterior pituitary gland focusing on lactotroph function. J Endocrinol 2018 Oct 1.

31. Filardo EJ, Thomas P. Minireview: G proteincoupled estrogen receptor-1, GPER-1: its mechanism of action and role in female reproductive cancer, renal and vascular physiology. Endocrinology 2012;153:2953-62.

32. Feldman RD. Heart Disease in Women: Unappreciated Challenges, GPER as a New Target. Int $\mathrm{J}$ Mol Sci 2016;17. 
33. Li WL, Xiang W, Ping Y. Activation of novel estrogen receptor GPER results in inhibition of cardiocyte apoptosis and cardioprotection. Mol Med Rep 2015;12:2425-30.

34. Feldman RD, Gros R, Ding Q, Hussain Y, Ban MR, McIntyre AD, et al. A common hypofunctional genetic variant of GPER is associated with increased blood pressure in women. $\mathrm{Br} \mathrm{J}$ Clin Pharmacol 2014;78:1441-52.

35. Santollo J, Daniels D. Activation of G protein-coupled estrogen receptor 1 (GPER-1) decreases fluid intake in female rats. Horm Behav 2015;73:39-46.

36. Li Z, Cheng L, Liang H, Duan W, Hu J, Zhi W, et al.
GPER inhibits diabetes-mediated RhoA activation to prevent vascular endothelial dysfunction. Eur J Cell Biol 2016;95:100-13.

37. Lu CL, Herndon C. New roles for neuronal estrogen receptors. Neurogastroenterol Motil 2017;29.

38. Wang J, Yu R, Han QQ, Huang HJ, Wang YL, Li HY, et al. G-1 exhibit antidepressant effect, increase of hippocampal ERs expression and improve hippocampal redox status in aged female rats. Behav Brain Res 2019;359:845-52.

39. Borrow AP, Handa RJ. Estrogen receptors modulation of anxiety-like behavior. Vitam Horm 2017;103:27-52. 